\title{
Rapid stereoselective access to the tetracyclic core of puupehenone and related sponge metabolites using metal-free radical cyclisations of cyclohexenyl-substituted 3-bromochroman-4-ones
}

\author{
Robin G. Pritchard, Helen M. Sheldrake, Isobel Z. Taylor, Timothy W. Wallace* \\ School of Chemistry, The University of Manchester, Oxford Road, Manchester M13 9PL, UK
}

\section{A R T I C L E I N F O}

\section{Article history:}

Received 26 March 2008

Accepted 21 April 2008

Available online 24 April 2008

\section{Keywords:}

Sesquiterpene-phenol

Merosesquiterpene

Benzo[a]xanthene

Puupehenone

Radical cyclisation

6-endo-Trig

$\alpha$-Bromoketone

Lauroyl peroxide

\begin{abstract}
A B S T R A C T
The tetracyclic nucleus of puupehenone, 15-oxopuupehenol and other sesquiterpene-phenol natural products can be assembled stereoselectively in three steps, the last of these being the 6-endo-trig cyclisation of an alpha-keto radical generated from a substituted 2-(2-cyclohexenyl)ethyl 3-bromo-4-chromanone under metal-free conditions.
\end{abstract}

(c) 2008 Elsevier Ltd. All rights reserved.
Marine organisms are an important resource in the search for molecules of therapeutic value, providing a steady stream of novel structures with potentially useful biological activity. ${ }^{1}$ One particular series in this category is based on a fusion of sesquiterpene and phenolic moieties, and includes the puupehenones 1-3, whose isolation from a sponge later identified as Heteronema sp. was reported in 1979., ${ }^{2,3}$ The parent compound (+)-puupehenone 1 has since been isolated from other sponges, mainly of the orders Verongida and Dictyoceratida, ${ }^{4-11}$ often accompanied by derived dimers. ${ }^{4,6 b, 9}$ Other members of the series include the formal 1,6addition products $4^{6}$ and 5 , $^{8 \mathrm{~b}}(-)-8$-epi-chromazonarol $6{ }^{12}(-)$ 15-oxopuupehenol 7,,11 the dehydro systems $\mathbf{8}^{7}$ and $\mathbf{9},{ }^{9}(+)-$ puupehedione $\mathbf{1 0}^{6 \mathrm{~b}}$ and (-)-kampanol A 11, which, exceptionally, is a fungal metabolite of terrestrial origin. ${ }^{13} \mathrm{~A}$ diastereoisomeric series include both antipodes of chromazonarol $\mathbf{1 2}^{\mathbf{1 4}}$ as well as cyclospongiaquinone-1 $13^{15}$ and (+)-hongoquercin A $14 .^{16}$

The biological activity observed within this series is diverse, with antitumour, ${ }^{6-8}$ antimalarial, ${ }^{7,8 b}$ antiviral, ${ }^{6}$ antifungal, ${ }^{2,6 b, 8}$ antibacterial, ${ }^{2,6 a, 8,16}$ antituberculosis ${ }^{17}$ and immunomodulatory ${ }^{6 \mathrm{~b}}$ properties having been reported. Additionally, compounds 1, 2 and $\mathbf{9}$ are lipoxygenase inhibitors, ${ }^{10 a}$ various derivatives of puupehedione 10, especially the synthetic (+)-8-epi-puupehedione $\mathbf{1 5},{ }^{18}$ inhibit angiogenesis, ${ }^{19}$ and (-)-kampanol A 11 is a specific

\footnotetext{
* Corresponding author. Fax: +4401612754598.

E-mail address: tim.wallace@manchester.ac.uk (T. W. Wallace).
}

inhibitor of Ras protein farnesyltransferase, making it a potential lead compound for new anticancer agents. ${ }^{13 \mathrm{~b}}$ The electrophilic nature of puupehenone $\mathbf{1}$ has been exploited in the formation of antigenic peptide conjugates for use in tumour immunotherapy studies. $^{20}$

The widespread interest in these structures has prompted various studies directed towards their synthesis. A route to $( \pm)-\mathbf{1}$ from farnesyl bromide and sesamol was reported by Trammell in $1978,{ }^{21}$ the key step being an acid-mediated stereoselective closure of the C-ring ( $41 \%$ de). Homochiral materials have generally been obtained by the elaboration of commercial sesquiterpenes such as (-)-sclareol 16, (+)-manool 17 or (+)-sclareolide 18, ${ }^{18,22}$ although a formal synthesis of $\mathbf{1 0}$ and $\mathbf{1 5}$ from $(R)-(-)$-carvone has also been described. ${ }^{23}$ In alternative approaches to this series based on polyene cyclisations, Yamamoto and coworkers developed enantioselective routes to (-)-chromazonarol 12 and (+)-8epi-puupehedione 15 from aryl polyenes, ${ }^{24}( \pm)$-hongoquercin $\mathrm{A}$ 14 was obtained from a geranylated chromene derivative, ${ }^{25}$ and titanocene-catalysed radical cyclisations of epoxypolyenes were used in formal syntheses of $( \pm)-\mathbf{1 0}$ and $( \pm)-\mathbf{1 5} .^{26}$

Our interest in this series and the need for new antimalarials led us to focus on the tetracyclic benzo[a]xanthen-12-one nucleus of 15-oxopuupehenol 7, whose functionality makes it potentially useful as a precursor of other members of the series and a variety of synthetic analogues. We established that a $C(4 a)-C(12 a)$ lactonebridged variant of this tetracyclic nucleus could be assembled in 
<smiles>[R]C1=C(O)C(=O)C=C2O[C@@]3(C)CC[C@H]4C(C)(C)CCC[C@]4(C)[C@]3(C)C=C21</smiles><smiles>[R]c1cc2c(cc1O)[C@@H]([X])[C@@H]1[C@](C)(CC[C@H]3C(C)(C)CCC[C@@]31C)O2</smiles>

$1 \mathrm{R}=\mathrm{H}$

$2 \mathrm{R}=\mathrm{Cl}$

$3 \mathrm{R}=\mathrm{Br}$

$4 \mathrm{R}=\mathrm{OH}, \mathrm{X}=\mathrm{CN}$

$5 \mathrm{R}=\mathrm{OH}, \mathrm{X}=\mathrm{OMe}$ $6 \mathrm{R}=\mathrm{X}=\mathrm{H}$<smiles>CC1(C)CCC[C@]2(C)[C@H]3CC[C@]4(C)Oc5cc(O)c(O)cc5C(=O)[C@]4(C)[C@H]3CC[C@]12C</smiles>

7<smiles>CC1(C)CCC[C@]2(C)[C@@H]3Cc4cc(O)ccc4O[C@@H]3CC[C@]12C</smiles>

12<smiles>COC1=CC(=O)C2=C(CC3[C@](C)(CC[C@@H]4C(C)(C)CCC[C@]34C)O2)C1=O</smiles>

13<smiles>[X]c1c(O)c(O)cc2c1C=C1[C@](C)(CC[C@H]3C(C)(C)CCC[C@@]13C)O2</smiles>

$8 \mathrm{R}=\mathrm{H}, \mathrm{X}=\mathrm{Cl}$ $9 \mathrm{R}=\mathrm{Me}, \mathrm{X}=\mathrm{H}$<smiles>Cc1cc2c(c(O)c1C(=O)O)C[C@H]1[C@](C)(CCC3C(C)(C)CCC[C@@]31C)O2</smiles>

14<smiles>CC1(C)CCC[C@]2(C)C3=CC4=CC(=O)C(=O)C=C4O[C@]3(C)CC[C@@H]12</smiles>

10<smiles>CC1(C)CCC[C@]2(C)C3=CC4=CC(=O)C(=O)C=C4O[C@]3(C)CC[C@H]12</smiles>

15<smiles>C=C[C@](C)(O)CC[C@H]1[C@@](C)(O)CC[C@H]2C(C)(C)CCC[C@@]21C</smiles>

16<smiles>C=C[C@](C)(O)CC[C@H]1C(=C)CC[C@H]2C(C)(C)CCC[C@]12C</smiles>

17<smiles>CC1(C)CCC[C@@]2(C)[C@@H]1CC[C@]1(C)OC(=O)C[C@@H]12</smiles>

18 four steps via a manganese(III) acetate-mediated oxidative radical cyclisation, ${ }^{27}$ and now wish to describe a novel metal-free variant of this cyclisation, which provides the functionalised tetracyclic nucleus in only three steps from commercial materials.

Our method for assembling the desired tetracycle is illustrated in Scheme 1 . Condensing dihydro- $\beta$-ionone 19 with the acetophenone 20a using pyrrolidine in toluene ${ }^{28}$ gave the chroman-4-one $( \pm)-\mathbf{2 1 a}{ }^{29}$ which could be brominated efficiently and selectively at $\mathrm{C}(3)$ using copper(II) bromide in ethyl acetate-chloroform ${ }^{30}$ to produce a diastereoisomeric mixture of bromoketones 22a (ratio ca. 3:2 by ${ }^{1} \mathrm{H}$ NMR spectroscopy). ${ }^{29}$ Heating the mixed bromoketones 22a with two equivalents of dilauroyl peroxide in refluxing 1,2-dichloroethane gave a mixture in which the major product was identified as the tetracyclic ketone 23a (56\%) on the basis of its mass spectrum $\left(\mathrm{MH}^{+} 311\right)$ and distinctive ${ }^{1} \mathrm{H}$ NMR signals at $\delta$ $5.57 \mathrm{ppm}$ (triplet, $J 3.8 \mathrm{~Hz}$ ) and 2.8-2.4 ppm (two double doublets, $J 3.8$ and $19.7 \mathrm{~Hz}$ ), which could be attributed to the isolated spin system composed of the three hydrogens attached to $C(5)$ and $\mathrm{C}(6){ }^{29}$

Repeating the sequence with the acetophenone $\mathbf{2 0 b}$ as the starting material gave good yields of the expected intermediates $\mathbf{2 1 b}$ and $\mathbf{2 2} \mathbf{b}$, and heating the latter with lauroyl peroxide gave the tetracyclic ketone $\mathbf{2 3 b}$ in $67 \%$ yield, together with some unreacted bromoketone 22b (13\%). ${ }^{31}$ Comparison of the respective ${ }^{1} \mathrm{H}$ and ${ }^{13} \mathrm{C}$ NMR spectra indicated that the structures of $\mathbf{2 3} \mathbf{a}$ and $\mathbf{2 3 b}$ were analogous. The relative stereochemistry of ketone $\mathbf{2 3 b}$ was confirmed by X-ray crystallography (Fig. 1). ${ }^{32}$

Mechanistically the transformation of 22a into 23a can be interpreted in terms of the initial abstraction of the bromine atom from<smiles>CC(=O)CCC1=C(C)CCCC1(C)C</smiles><smiles>[R]c1cc(O)c(C(C)=O)cc1[R9]</smiles>

20

a $\mathrm{R}=\mathrm{H}$

b $\mathrm{R}=\mathrm{OMe}$<smiles>[R]c1cc2c(cc1[R])[C@@]1(C)C(=O)c3cc([R])c([R])cc3[C@H]3CCCC(C)(C)C3=CC[C@@]1(C)O2</smiles>

23<smiles>[R]c1cc2c(cc1[R])C(=O)CC(C)(CCC1=C(C)CCCC1(C)C)O2</smiles>

21
ii $\mid \begin{aligned} & \text { a 90\% } \\ & \text { b 99\% }\end{aligned}$

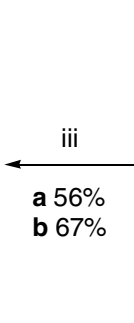

22

Scheme 1. Reagents and conditions: (i) pyrrolidine, toluene, reflux (Dean-Stark), $3 \mathrm{~d}$; (ii) $\mathrm{CuBr}_{2}$, EtOAc- $\mathrm{CHCl}_{3}$, reflux, 5-14 h; (iii) lauroyl peroxide 24 (2.0 equiv), 1,2dichloroethane, reflux, 6-21 h.

22a by the undecanyl radical $\mathbf{2 6}$, the latter being generated via the thermolysis of lauroyl peroxide $\mathbf{2 4}$ (Scheme 2). Related processes involving the abstraction of iodine have been described by Renaud and co-workers, ${ }^{33}$ but we are not aware of any bromoketones being used in this way. The presence of 1-bromoundecane $\mathbf{2 7}$ among the by-products of the reaction was confirmed by ${ }^{1} \mathrm{H}$ NMR spectroscopy. The 6-endo-trig cyclisation of $\mathbf{2 8}$, leading to 29 in which the $C(6 a)$ and $C(12 b)$ methyl groups have a trans relationship, is consistent with our earlier observation of this type of 


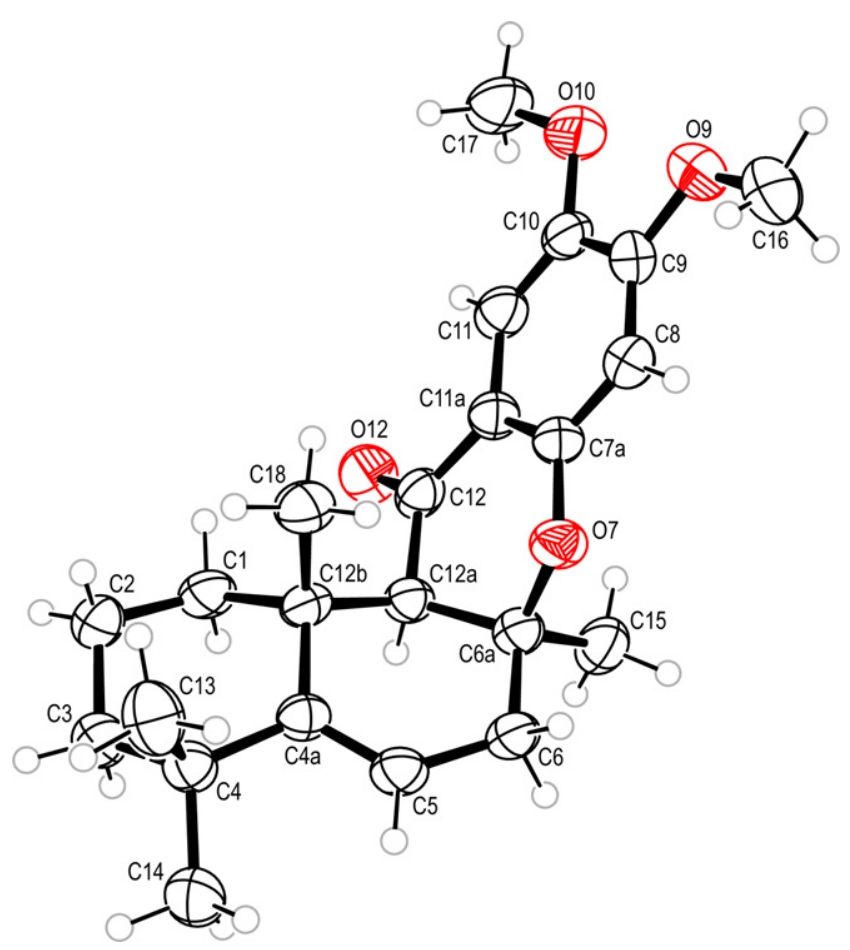

Figure 1. X-ray structure of 23b generated using ORTEP (thermal ellipsoids 50\%).

ring closure ${ }^{27}$ and the stereoselection is assumed to be conformationally driven. The pathways linking the radical $\mathbf{2 9}$ with the final product 23a are open to speculation. Lauroyl peroxide can participate in one-electron oxidations ${ }^{34}$ and the cation $\mathbf{3 0}$ is presumed to be the immediate precursor of the alkene 23a, but it is unclear whether the laurate ester $\mathbf{3 3}$ is involved. Lauric acid $\mathbf{3 2}$ was also identified among the by-products of the reaction by ${ }^{1} \mathrm{H}$ NMR spectroscopy.

In summary, the tetracyclic nucleus of puupehenone, 15oxopuupehenol and related sesquiterpene-phenol natural products can be assembled stereoselectively in three steps, the last of these being the peroxide-induced 6-endo-trig cyclisation of an alpha-keto radical generated from a substituted 2-(2-cyclohexenyl)ethyl 3-bromo-4-chromanone. The cyclisation process is terminated oxidatively with the formation of an olefinic bond, which should render the tetracyclic system amenable to further elaboration. We are currently exploring this possibility and various mechanistic aspects of the cyclisation reaction.

\section{Acknowledgements}

We are grateful to the EPSRC for their financial support of this work, and we thank Val Boote and Steve Kelly for assistance with MS and NMR measurements. We also wish to acknowledge the use of the EPSRC's Chemical Database Service at Daresbury. ${ }^{35}$

\section{Supplementary data}

X-ray crystallographic data for $\mathbf{2 3 b}$. Supplementary data associated with this article can be found, in the online version, at doi:10.1016/j.tetlet.2008.04.114.

\section{References and notes}

1. For reviews, see (a) Blunt, J. W.; Copp, B. R.; Hu, W.-P.; Munro, M. H. G.; Northcote, P. T.; Prinsep, M. R. Nat. Prod. Rep. 2008, 25, 35-94; (b) Morris, J. C.; Phillips, A. J. Nat. Prod. Rep. 2008, 25, 95-117; (c) Lebar, M. D.; Heimbegner, J. L. Baker, B. J. Nat. Prod. Rep. 2007, 24, 774-797; (d) Bowling, J. J.; Kochanowska, A. J.; Kasanah, N.; Hamann, M. T. Exp. Opin. Drug Discovery 2007, 2, 15051522.

2. Ravi, B. N.; Perzanowski, H. P.; Ross, R. A.; Erdman, T. R.; Scheuer, P. J.; Finer, J.; Clardy, J. Pure Appl. Chem. 1979, 51, 1893-1900.

3. Urban, S.; Capon, R. J. J. Nat. Prod. 1996, 59, 900-901.

4. Amade, P.; Chevelot, L.; Perzanowski, H. P.; Scheuer, P. J. Helv. Chim. Acta 1983, $66,1672-1675$

5. Kohmoto, S.; McConnell, O. J.; Wright, A.; Koehn, F.; Thompson, W.; Lui, M.; Snader, K. M. J. Nat. Prod. 1987, 50, 336.

6. (a) Hamann, M. T.; Scheuer, P. J. Tetrahedron Lett. 1991, 32, 5671-5672; (b) Hamann, M. T.; Scheuer, P. J.; Kelly-Borges, M. J. Org. Chem. 1993, 58, 65656569.

7. Nasu, S. S.; Yeung, B. K. S.; Hamann, M. T.; Scheuer, P. J.; Kelly-Borges, M.; Goins, K. J. Org. Chem. 1995, 60, 7290-7292.

8. (a) Bourguet-Kondracki, M.-L.; Debitus, C.; Guyot, M. Tetrahedron Lett. 1996, 37 3861-3864; (b) Bourguet-Kondracki, M.-L.; Lacombe, F.; Guyot, M. J. Nat. Prod. 1999, 62, 1304-1305.

9. Piña, I. C.; Sanders, M. L.; Crews, P. J. Nat. Prod. 2003, 66, 2-6.

10. (a) Amagata, T.; Whitman, S.; Johnson, T. A.; Stessman, C. C.; Loo, C. P.; Lobkovsky, E.; Clardy, J.; Crews, P.; Holman, T. R. J. Nat. Prod. 2003, 66, 230235; (b) Ciavatta, M. L.; Lopez Gresa, M. P.; Gavagnin, M.; Romero, V.; Melck, D.; Manzo, E.; Guo, Y.-W.; van Soest, R.; Cimino, G. Tetrahedron 2007, 63, 13801384.

11. Ueda, K.; Ogi, T.; Sato, A.; Siwu, E. R. O.; Kita, M.; Uemura, D. Heterocycles 2007 72, 655-663.

12. Djura, P.; Stierle, D. B.; Sullivan, B.; Faulkner, D. J.; Arnold, E.; Clardy, J. J. Org. Chem. 1980, 45, 1435-1441.

13. (a) Singh, S. B.; Zink, D. L.; Williams, M.; Polishook, J. D.; Sanchez, M.; Silverman, K. C.; Lingham, R. B. Bioorg. Med. Chem. Lett. 1998, 8, 2071-2076; (b) Iwasaki, K.; Nakatani, M.; Inoue, M.; Katoh, T. Tetrahedron 2003, 59, 87638773.

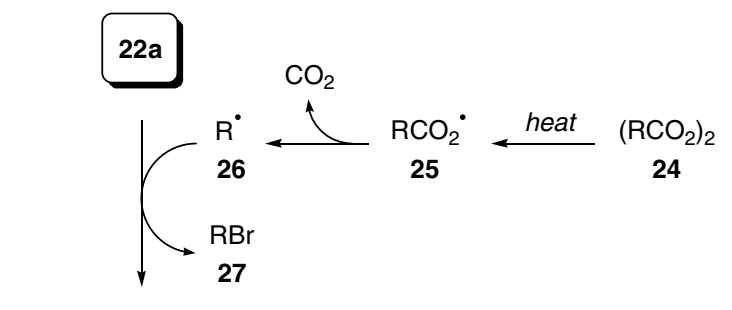<smiles>CC1=C(CCC2(C)CC(=O)c3ccccc3O2)CCCC1(C)C</smiles>

28

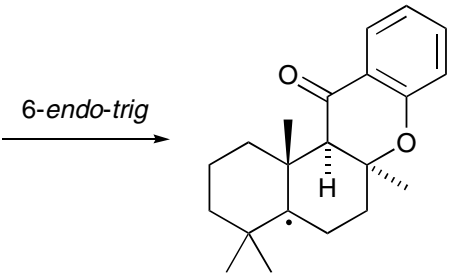

29
$23 a$<smiles>CC(C)CCCC(=O)O</smiles>

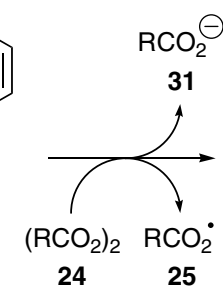<smiles>CC1(C)CCC[C@]2(C)[C@H]3CC[C@]4(C)Oc5ccccc5C(=O)[C@]4(C)[C@H]3CC[C@]12C</smiles>

30<smiles>CC1(C)CCCC2(C)C1CCC1(C)Oc3ccccc3C(=O)C12C(=O)O</smiles>

33

Scheme 2. A possible mechanistic rationale for the formation of 23a from the bromoketone 22a. 
14. (a) Fenical, W.; McConnell, O. Experientia 1975, 31, 1004-1005; (b) Cimino, G. De Stefano, S.; Minale, L. Experientia 1975, 31, 1117-1118; (c) Song, F.; Fan, X: Xu, X.; Zhao, J.; Yang, Y.; Shi, J. J. Nat. Prod. 2004, 67, 1644-1649.

15. (a) Kazlauskas, R.; Murphy, P. T.; Warren, R. G.; Wells, R. J.; Blount, J. F. Aust. J. Chem. 1978, 31, 2685-2697; (b) Capon, R. J.; Groves, D. R.; Urban, S.; Watson, R. G. Aust. J. Chem. 1993, 46, 1245-1253; (c) Jankam, A.; Somerville, M. J.; Hooper, J. N. A.; Brecknell, D. J.; Suksamrarn, A.; Garson, M. J. Tetrahedron 2007, 63, 1577-1582.

16. (a) Roll, D. M.; Manning, J. K.; Carter, G. T. J. Antibiot. 1998, 51, 635-639; (b) Abbanat, D. A.; Singh, M. P.; Greenstein, M. J. Antibiot. 1998, 51, 708-714; (c) Tsujimori, H.; Bando, M.; Mori, K. Eur. J. Org. Chem. 2000, 297-302.

17. El Sayed, K. A.; Bartyzel, P.; Shen, X.; Perry, T. L.; Zjawiony, J. K.; Hamann, M. T. Tetrahedron 2000, 56, 949-953.

18. Barrero, A. F.; Alvarez-Manzaneda, E. J.; Chahboun, R.; Cortés, M.; Armstrong, V. Tetrahedron 1999, 55, 15181-15208.

19. Castro, M. E.; Gonzalez-Iriarte, M.; Barrero, A. F.; Salvador-Tormo, N Munoz-Chapuli, R.; Medina, M. A.; Quesada, A. R. Int. J. Cancer 2004, 110, 3138.

20. Douat-Casassus, C.; Marchand-Geneste, N.; Diez, E.; Aznar, C.; Picard, P.; Geoffre, S.; Huet, A.; Bourguet-Kondracki, M.-L.; Gervois, N.; Jotereau, F.; Quideau, S. Mol. BioSyst. 2006, 2, 240-249.

21. Trammell, G. L. Tetrahedron Lett. 1978, 1525-1528.

22. (a) Barrero, A. F.; Alvarez-Manzaneda, E. J.; Chahboun, R. Tetrahedron Lett. 1997, 38, 2325-2328; (b) Arjona, O.; Garranzo, M.; Mahugo, J.; Maroto, E.; Plumet, J.; Sáez, B. Tetrahedron Lett. 1997, 38, 7249-7252; Arjona, O.; Garranzo, M.; Mahugo, J.; Maroto, E.; Plumet, J.; Sáez, B. Tetrahedron Lett. 1997, 38, 8605; (c) Barrero, A. F.; Alvarez-Manzaneda, E. J.; Herrador, M. M.; Chahboun, R.; Galera, P. Bioorg. Med. Chem. Lett. 1999, 9, 2325-2328; (d) Quideau, S.; Lebon, M.; Lamidey, A.-M. Org. Lett. 2002, 4, 3975-3978; (e) Armstrong, V.; Barrero, A. F.; Alvarez-Manzaneda, E. J.; Cortés, M.; Sepúlveda, B. J. Nat. Prod. 2003, 66, 1382 1383; (f) Hua, D. H.; Huang, X.; Chen, Y.; Battina, S. K.; Tamura, M.; Noh, S. K. Koo, S. I.; Namatame, I.; Tomoda, H.; Perchellet, E. M.; Perchellet, J.-P. J. Org Chem. 2004, 69, 6065-6078; (g) Alvarez-Manzaneda, E. J.; Chahboun, R. Barranco Pérez, I.; Cabrera, E.; Alvarez, E.; Alvarez-Manzaneda, R. Org. Lett. 2005, 7, 1477-1480; (h) Villamizar, J.; Plata, F.; Canudas, N.; Tropper, E. Fuentes, J.; Orcajo, A. Synth. Commun. 2006, 36, 311-320; (i) Alvarez Manzaneda, E. J.; Chahboun, R.; Cabrera, E.; Alvarez, E.; Haidour, A.; Ramos, J. M.; Alvarez-Manzaneda, R.; Hmamouchi, M.; Bouanou, H. J. Org. Chem. 2007, 72, 3332-3339; (j) Quideau, S.; Pouységu, L.; Deffieux, D. Synlett 2008, 467495.

23. Maiti, S.; Sengupta, S.; Giri, C.; Achari, B.; Banerjee, K. K. Tetrahedron Lett. 2001 42, 2389-2391.

24. (a) Ishihara, K.; Nakamura, S.; Yamamoto, H. J. Am. Chem. Soc. 1999, 121, $4906-$ 4907; (b) Nakamura, S.; Ishihara, K.; Yamamoto, H. J. Am. Chem. Soc. 2000, 122 8131-8140; (c) Ishibashi, H.; Ishihara, K.; Yamamoto, H. J. Am. Chem. Soc. 2004 126, 11122-11123.

25. Kurdyumov, A. V.; Hsung, R. P. J. Am. Chem. Soc. 2006, 128, 6272-6273.

26. (a) Gansäuer, A.; Rosales, A.; Justicia, J. Synlett 2006, 927-929; (b) Gansäuer, A.; Justicia, J.; Rosales, A.; Worgull, D.; Rinker, B.; Cuerva, J. M.; Oltra, J. E. Eur. J Org. Chem. 2006, 4115-4127.

27. Crombie, B. S.; Smith, C.; Varnavas, C. Z.; Wallace, T. W. J. Chem. Soc., Perkin Trans. 1 2001, 206-215.

28. Method: Kabbe, H.-J.; Widdig, A. Angew. Chem., Int. Ed. Engl. 1982, 21, 247-256.

29. Selected data: 21a, $\tan$ oil $\left(\mathrm{MH}^{+}, 313.2177 ; \mathrm{C}_{21} \mathrm{H}_{29} \mathrm{O}_{2}\right.$ requires 313.2168); $v_{\max }$ $\mathrm{cm}^{-1}\left(\mathrm{CHCl}_{3}\right) 1684 ; \delta_{\mathrm{H}}\left(400 \mathrm{MHz}, \mathrm{CDCl}_{3}\right) 7.85(1 \mathrm{H}, \mathrm{dd}, J 1.7,8.0 \mathrm{~Hz}, 5-\mathrm{H}), 7.46$ $(1 \mathrm{H}$, ddd, $J 1.7,7.0,8.0 \mathrm{~Hz}, 7-\mathrm{H}), 6.96(1 \mathrm{H}$, apparent t, $J$ ca. $7.5 \mathrm{~Hz}, 6-\mathrm{H}), 6.95(1 \mathrm{H}$ d, $J 8.0 \mathrm{~Hz}, 8-\mathrm{H}), 2.79(1 \mathrm{H}, \mathrm{d}, J 16.4 \mathrm{~Hz}, 3-\mathrm{H}), 2.72(1 \mathrm{H}, \mathrm{d}, J 16.4 \mathrm{~Hz}, 3-\mathrm{H}), 2.20$ $2.07\left(2 \mathrm{H}, \mathrm{m}, 4^{\prime \prime}-\mathrm{H}_{2}\right), 1.91-1.82\left(3 \mathrm{H}, \mathrm{m}, 1^{\prime}-\mathrm{H}\right.$ and $\left.3^{\prime \prime}-\mathrm{H}_{2}\right), 1.76-1.67\left(1 \mathrm{H}, \mathrm{m}, 1^{\prime}-\mathrm{H}\right)$ $1.58-1.50\left(2 \mathrm{H}, \mathrm{m}, 2^{\prime}-\mathrm{H}_{2}\right), 1.47$ (3H, s, $\left.2^{\prime \prime}-\mathrm{Me}\right), 1.465$ (3H, s, 2-Me), 1.40-1.35 $\left(2 \mathrm{H}, \mathrm{m}, 5^{\prime \prime}-\mathrm{H}_{2}\right), 0.97\left(3 \mathrm{H}, \mathrm{s}, 6^{\prime \prime}-\mathrm{Me}\right), 0.88\left(3 \mathrm{H}, \mathrm{s}, 6^{\prime \prime}-\mathrm{Me}\right) ; R_{\mathrm{f}} 0.56$ (hexane-EtOAc 3:1). Compound 21b, tan oil $\left(\mathrm{MH}^{+}, 373.2388 ; \mathrm{C}_{23} \mathrm{H}_{33} \mathrm{O}_{4}\right.$ requires 373.2379); $v_{\max } / \mathrm{cm}^{-1}\left(\mathrm{CHCl}_{3}\right) 1673 ; \delta_{\mathrm{H}}\left(300 \mathrm{MHz}, \mathrm{CDCl}_{3}\right) 7.23(1 \mathrm{H}, \mathrm{s}, 5-\mathrm{H}), 6.39(1 \mathrm{H}, \mathrm{s}, 8-$ $\mathrm{H}), 3.88(3 \mathrm{H}, \mathrm{s}, \mathrm{OMe}), 3.84(3 \mathrm{H}, \mathrm{s}, \mathrm{OMe}), 2.71(1 \mathrm{H}, \mathrm{d}, J 16.5 \mathrm{~Hz}, 3-\mathrm{H}), 2.62(1 \mathrm{H}, \mathrm{d}$
$16.5 \mathrm{~Hz}, 3-\mathrm{H}), 2.15-2.02\left(2 \mathrm{H}, \mathrm{m}, 4^{\prime \prime}-\mathrm{H}_{2}\right), 1.90-1.78\left(3 \mathrm{H}, \mathrm{m}, 1^{\prime}-\mathrm{H}\right.$ and $\left.3^{\prime \prime}-\mathrm{H}_{2}\right)$ $1.74-1.61\left(1 \mathrm{H}, \mathrm{m}, 1^{\prime}-\mathrm{H}\right), 1.55-1.47\left(2 \mathrm{H}, \mathrm{m}, 2^{\prime}-\mathrm{H}_{2}\right), 1.46\left(3 \mathrm{H}, \mathrm{s}, 2^{\prime \prime}-\mathrm{Me}\right), 1.42(3 \mathrm{H}$, s, 2-Me), $1.40-1.32\left(2 \mathrm{H}, \mathrm{m}, 5^{\prime \prime}-\mathrm{H}_{2}\right), 0.94\left(3 \mathrm{H}, \mathrm{s}, 6^{\prime \prime}-\mathrm{Me}\right), 0.87$ (3H, s, 6"-Me); $R_{\mathrm{f}}$ 0.52 (hexane-EtOAc, 7:3). Compound 22a, yellow oil; $v_{\max } / \mathrm{cm}^{-1}\left(\mathrm{CHCl}_{3}\right) 1704$; $\delta_{\mathrm{H}}\left(400 \mathrm{MHz}, \mathrm{CDCl}_{3}\right)$ (major isomer) $7.89(1 \mathrm{H}, \mathrm{dd}, J 1.7,7.8 \mathrm{~Hz}, 5-\mathrm{H}), 7.53(1 \mathrm{H}$, ddd, J 1.7, 7.2, 8.3 Hz, 7-H), 7.10-6.95 (2H, m, 6-H, 8-H), 4.46 (1H, s, 3-H), 2.24$1.78\left(4 \mathrm{H}, \mathrm{m}, 2^{\prime}-\mathrm{H}_{2}, 3^{\prime \prime}-\mathrm{H}_{2}\right), 1.70-1.30\left(6 \mathrm{H}, \mathrm{m}, 1^{\prime}-\mathrm{H}_{2}, 4^{\prime \prime}-\mathrm{CH}_{2}, 5^{\prime \prime}-\mathrm{CH}_{2}\right), 1.61$ (3H, s, $2^{\prime \prime}-\mathrm{Me}$ ), 1.33 (3H, s, 2-Me), 0.91 (3H, s, 6" -Me), 0.76 (3H, s, 6"-Me); (minor isomer) $4.42(1 \mathrm{H}, \mathrm{s}, 3-\mathrm{H}), 1.61\left(3 \mathrm{H}, \mathrm{s}, 2^{\prime \prime}-\mathrm{Me}\right), 1.54(3 \mathrm{H}, \mathrm{s}, 2-\mathrm{Me}), 1.02\left(3 \mathrm{H}, \mathrm{s}, 6^{\prime \prime}-\right.$ Me), 0.96 ( $3 \mathrm{H}, \mathrm{s}, 6^{\prime \prime}-\mathrm{Me}$ ); $R_{\mathrm{f}}$ 0.48, 0.41 (hexane-EtOAc, 5:1). Compound 22b, dark yellow oil; $v_{\max } / \mathrm{cm}^{-1}\left(\mathrm{CHCl}_{3}\right) 1680 ; \delta_{\mathrm{H}}\left(400 \mathrm{MHz}, \mathrm{CDCl}_{3}\right)$ (major isomer) $7.28(1 \mathrm{H}, \mathrm{s}, 5-\mathrm{H}), 6.47(1 \mathrm{H}, \mathrm{s}, 8-\mathrm{H}), 4.39(1 \mathrm{H}, \mathrm{s}, 3-\mathrm{H}), 3.92(3 \mathrm{H}, \mathrm{s}, \mathrm{OMe}), 3.88(3 \mathrm{H}$, s, OMe), 2.20-1.75 (4H, m, 2'- $\left.\mathrm{H}_{2}, 3^{\prime \prime}-\mathrm{H}_{2}\right), 1.65-1.20\left(6 \mathrm{H}, \mathrm{m}, 1^{\prime}-\mathrm{H}_{2}, 4^{\prime \prime}-\mathrm{CH}_{2}, 5^{\prime \prime}-\right.$ $\mathrm{CH}_{2}$ ), 1.61 (3H, s, 2"-Me), 1.35 (3H, s, 2-Me), 0.91 (3H, s, 6"-Me), 0.79 (3H, s, 6" $\mathrm{Me})$;(minor isomer) $6.44(1 \mathrm{H}, \mathrm{s}, 8-\mathrm{H}), 4.35(1 \mathrm{H}, \mathrm{s}, 3-\mathrm{H}), 1.61$ (3H, s, 2"-Me), 1.54 $(3 \mathrm{H}, \mathrm{s}, 2-\mathrm{Me}), 1.04$ (3H, s, 6"-Me) 0.99 ( $\left.3 \mathrm{H}, \mathrm{s}, 6^{\prime \prime}-\mathrm{Me}\right) ; R_{\mathrm{f}} 0.26,0.21$ (hexaneEtOAc, 3:1). Compound 23a, pale yellow waxy oil $\left(M^{+}\right.$, 311.2016; $\mathrm{C}_{21} \mathrm{H}_{27} \mathrm{O}_{2}$ requires 311.2011); $v_{\max } / \mathrm{cm}^{-1}\left(\mathrm{CHCl}_{3}\right) 1680 ; \delta_{\mathrm{H}}\left(300 \mathrm{MHz}, \mathrm{CDCl}_{3}\right) 7.86(1 \mathrm{H}$, dd, $J 1.7,7.8 \mathrm{~Hz}, 5-\mathrm{H}), 7.43(1 \mathrm{H}$, ddd, $J 1.7,7.1,8.3 \mathrm{~Hz}, 7-\mathrm{H}), 6.95(1 \mathrm{H}$, apparent $\mathrm{t}$, ca. $7.5 \mathrm{~Hz}, 6-\mathrm{H}), 6.87(1 \mathrm{H}, \mathrm{d}, J 8.3 \mathrm{~Hz}, 8-\mathrm{H}), 5.57(1 \mathrm{H}, \mathrm{t}, J 3.8 \mathrm{~Hz}, 5-\mathrm{H}), 2.72(1 \mathrm{H}$, dd, $J$ 3.8, $19.7 \mathrm{~Hz}, 6-\mathrm{H}), 2.47$ (1H, dd, $J 3.8,19.7 \mathrm{~Hz}, 6-\mathrm{H}), 2.19(1 \mathrm{H}, \mathrm{s}, 12 \mathrm{a}-\mathrm{H})$, $1.80-1.25\left(6 \mathrm{H}, \mathrm{m}, 1-\mathrm{CH}_{2}, 2-\mathrm{CH}_{2}, 3-\mathrm{CH}_{2}\right), 1.34$ (3H, s, 6a-Me), $1.14(3 \mathrm{H}, \mathrm{s}, 4-\mathrm{Me})$ $1.12(3 \mathrm{H}, \mathrm{s}, 4-\mathrm{Me}), 1.01$ (3H, s, 12b-Me); $R_{\mathrm{f}} 0.23$ (hexane-EtOAc, 3:1). Compound 23b, mp $165-166^{\circ} \mathrm{C}(\mathrm{EtOH})\left(\mathrm{MH}^{+}, 371.2213 ; \mathrm{C}_{23} \mathrm{H}_{31} \mathrm{O}_{4}\right.$ requires $371.2222) ; v_{\max } / \mathrm{cm}^{-1}\left(\mathrm{CHCl}_{3}\right) 1674 ; \delta_{\mathrm{H}}\left(400 \mathrm{MHz}, \mathrm{CDCl}_{3}\right) 7.28(1 \mathrm{H}, \mathrm{s}, 11-\mathrm{H})$, $6.37(1 \mathrm{H}, \mathrm{s}, 8-\mathrm{H}), 5.56(1 \mathrm{H}, \mathrm{t}, J 3.8 \mathrm{~Hz}, 5-\mathrm{H}), 3.89(6 \mathrm{H}, \mathrm{br} \mathrm{s}, 2 \mathrm{x} \mathrm{OMe}), 2.68(1 \mathrm{H}$, dd, $J 3.8,19.7 \mathrm{~Hz}, 6-\mathrm{H}), 2.47$ (1H, dd, $J 3.8,19.7 \mathrm{~Hz}, 6-\mathrm{H}), 2.13(1 \mathrm{H}, \mathrm{s}, 12 \mathrm{a}-\mathrm{H})$ $1.80-1.25\left(6 \mathrm{H}, \mathrm{m}, 1-\mathrm{CH}_{2}, 2-\mathrm{CH}_{2}, 3-\mathrm{CH}_{2}\right), 1.36(3 \mathrm{H}, \mathrm{s}, 6 \mathrm{a}-\mathrm{Me}), 1.14(3 \mathrm{H}, \mathrm{s}, 4-\mathrm{Me})$, $1.12\left(3 \mathrm{H}, \mathrm{s}, 4-\mathrm{Me}\right.$ ), 1.02 (3H, s, 12b-Me); $R_{\mathrm{f}} 0.27$ (hexane-EtOAc, 3:1).

30. King, L. C.; Ostrum, G. K. J. Org. Chem. 1964, 29, 3459-3461.

31. Procedure for the formation of 23b: A solution of the bromoketone $\mathbf{2 2 b}(0.520 \mathrm{~g}$, $1.15 \mathrm{mmol})$ in dry degassed 1,2-dichloroethane $(12 \mathrm{~mL})$ was heated to reflux for $15 \mathrm{~min}$. A solution of dilauroyl peroxide $(0.921 \mathrm{~g}, 2.31 \mathrm{mmol})$ in dry degassed 1,2-dichloroethane $(7 \mathrm{~mL})$ was added dropwise via a pressureequalising dropping funnel to the refluxing solution over $2.75 \mathrm{~h}$. After the completion of the addition, heating was continued overnight $(18.5 \mathrm{~h})$. The reaction mixture was cooled to room temperature and filtered through a pad of basic alumina $(5 \mathrm{~mm})$ on top of a pad of silica $(20 \mathrm{~mm})$, which was washed successively with EtOAc-hexane $(3: 1,80 \mathrm{~mL})$ and dichloromethane $(150 \mathrm{~mL})$. The combined filtrate was concentrated in vacuo and purified by flash column chromatography (EtOAc-hexane, 1:9), which gave recovered bromoketone 22b $(0.070 \mathrm{~g}, 13 \%)$ and the title compound $23 \mathrm{~b}(0.287 \mathrm{~g}, 67 \%, 78 \%$ based on recovered 22b) as a white solid.

32. Crystal data for 23b: Colourless crystals from EtOH, $\mathrm{C}_{23} \mathrm{H}_{30} \mathrm{O}_{4}, M=370.47$, orthorhombic, $\quad a=7.5154(2), \quad b=14.0755(5), \quad c=18.5551(6) \AA, \quad U=$ 1662.81(11) $\AA^{3}, T=293(2) \mathrm{K}$, space group $P 212121, Z=4, D_{\mathrm{c}}=1.254$ $\mathrm{Mg} \mathrm{m}^{-3}, \lambda\left(\mathrm{MoK}_{\alpha}\right)=0.71073 \AA, \mu=0.084 \mathrm{~mm}^{-1}, 14565$ reflections measured, 2197 unique $\left(R_{\mathrm{int}}=0.0874\right)$, which were used in all calculations. The final $w R\left(F^{2}\right)$ was 0.0907 (all data). Crystallographic data (excluding structure factors) for 23b have been deposited with the Cambridge Crystallographic Data Centre as supplementary publication number CCDC 679032. Copies of the data can be obtained, free of charge, on application to CCDC, 12 Union Road, Cambridge CB2 1EZ, UK (fax: +44-1223-336033 or e-mail: deposit@ccdc. cam.ac.uk).

33. (a) Ollivier, C.; Bark, T.; Renaud, P. Synthesis 2000, 1598-1602; (b) Kolly-Kovac, T.; Renaud, P. Synthesis 2005, 1459-1466.

34. (a) Coppa, F.; Fontana, F.; Minisci, F.; Pianese, G.; Tortoreto, P.; Zhao, L. Tetrahedron Lett. 1992, 33, 687-690; (b) Gagosz, F; Moutrille, C.; Zard, S. Z. Org. Lett. 2002, 4, 2707-2709; (c) Alajarín, M.; Vidal, A.; Ortín, M.-M. Org. Biomol. Chem. 2003, 1, 4282-4292.

35. Fletcher, D. A.; McMeeking, R. F.; Parkin, D. J. Chem. Inf. Comput. Sci. 1996, 36 746-749. 